\title{
Detection schemes for massive MIMO system with low-resolution ADCs
}

\author{
Peng Gao ${ }^{\text {a) }}$ and Yukitoshi Sanada ${ }^{\text {b) }}$ \\ Dept. of Electronics and Electrical Engineering, Keio University, \\ 3-14-1 Hiyoshi, Kohoku, Yokohama 223-8522, Japan \\ a)pgao@snd.elec.keio.ac.jp \\ b)sanada@elec.keio.ac.jp
}

\begin{abstract}
In a full-digital massive multi-user MIMO system, maximal-ratio combining (MRC) can obtain more considerable diversity gain while interstream interference (ISI) and multi-user interference (MUI) can be canceled using minimum mean square error (MMSE) algorithm. This letter evaluates the throughputs of detection schemes for different antenna numbers in the massive MIMO system with low-resolution analog-to-digital converters (ADCs). The letter makes a comparison between MRC and MMSE under quantization range limit. Numerical results show that MRC achieves better system performance with lower implementation complexity as the number of antennas increases.
\end{abstract}

Keywords: massive MIMO, low-resolution ADCs, MRC, MMSE, range limit

Classification: Transmission Systems and Transmission Equipment for Communications

\section{References}

[1] J. G. Andrews, S. Buzzi, W. Choi, S. V. Hanly, A. Lozano, A. C. K. Soong, and J. C. Zhang, "What will 5G be," IEEE J. Sel. Areas Commun., vol. 32, no. 6, pp. 1065-1082, June 2014. DOI:10.1109/JSAC.2014.2328098

[2] D. Dardari, "Joint clip and quantization effects characterization in OFDM receivers," IEEE Trans. Circuits Syst. I, Reg. Papers, vol. 53, no. 8, pp. 17411748, Aug. 2006. DOI:10.1109/TCSI.2006.875170

[3] S. Jacobsson, G. Durisi, M. Coldrey, U. Gustavsson, and C. Studer, "Throughput analysis of massive MIMO uplink with low-resolution ADCs," IEEE Trans. Wireless Commun., vol. 16, no. 6, pp. 4038-4051, June 2017. DOI:10.1109/ TWC.2017.2691318

[4] A. Azizzadeh, R. Mohammadkhani, and S. V. A. Makki, "BER performance of uplink massive MIMO with low-resolution ADCs," IEEE ICCKE, Oct. 2017. DOI:10.1109/ICCKE.2017.8167895

[5] T. E. Bogale and L. B. Le, "Beamforming for multiuser massive MIMO systems: Digital versus hybrid analog-digital," IEEE Global Communications Conference, Dec. 2014. DOI:10.1109/GLOCOM.2014.7037444

[6] S. Yoshioka, S. Suyama, T. Okuyama, J. Mashino, and Y. Okumura, "5G massive MIMO with digital beamforming and two-stage channel estimation for low SHF band," IEEE Wireless Days, Mar. 2017. DOI:10.1109/WD.2017.7918124 


\section{Introduction}

In the next generation mobile communication system, much higher area data throughput is required to manage the global demand for the continuously growing wireless data traffic [1]. It can achieve a multiple times larger bit rate by improving spectral efficiency (bits/s/Hz/cell) without the need for more bandwidth or additional base stations. Since a large number of the base station (BS) antennas effectively averages out noise and fading, and reduces the multi-user interference to a certain extent, massive MIMO is considered as a key technology.

Full digital massive MIMO deployment is difficult to realize because highresolution analog-to-digital converters (ADCs) produce primary power consumption. Owing to its favorable property of low cost and low power consumption, lowresolution ADCs (1 4 bits) have also been worth paying attention [2, 3, 4]. Reference [2] proves that an appropriate quantization range limit (clipping) can relieve the distortion in a single-input single-output OFDM system. In [3], the performance of single-carrier modulation in a massive MIMO system with lowresolution ADCs is analyzed while OFDM is assumed in this letter. In [4], it is shown that a minimum mean square error (MMSE) algorithm can achieve better BER using low-resolution ADCs in a hybrid analog-digital system with a large number of BS antennas. MMSE detection demands a large amount of computational complexity for matrix inversion while it achieves near-optimal performance. Maximal-ratio combing (MRC), as one beamforming scheme, can also be applied at the receiver of full-digital massive MIMO to achieve the maximum receive signal-to-interference-plus-noise ratio (SINR) [5].

In this letter, the achievable uplink rates of MRC and MMSE with the quantization range limit and with the different numbers of BS antennas are evaluated and compared. Residual multi-user interference (MUI) caused by lowresolution ADCs limits the system throughput even though MMSE is applied. On the other hand, when low-resolution ADCs are used, MRC achieves comparable throughput performance as that of MMSE. Thus, MRC is more suitable owing to its lower complexity.

\section{System model of uplink}

\subsection{Uplink system model}

The single-cell uplink system shown in Fig. 1 is assumed. We consider a fulldigital massive MIMO uplink with low-resolution ADCs. There are $K$ users, each

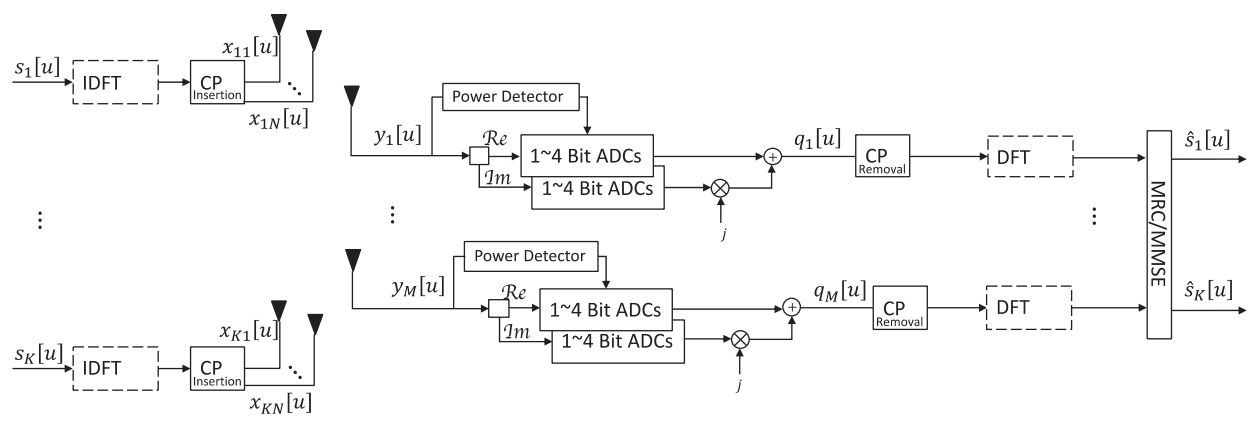

Fig. 1. Uplink system of massive MIMO with low-resolution ADCs. 
user has $N$ Tx antennas and one BS equipped with an array of $M$ antennas. Suppose that the size of an inverse discrete Fourier transform (IDFT) is $N_{d f t}$, and the signal from the $n$th antenna of the $k$ th user in the uplink is,

$$
s_{k n}[u]=\frac{1}{\sqrt{N_{d f t}}} \sum_{v=-N_{c p}}^{N_{d f f}-1} \mathrm{~S}_{k n}[v] e^{j 2 \pi u v / N_{d f t}},
$$

where $\mathrm{S}_{k n}[v]$ is the transmit signal from the $n$th antenna of the $k$ th user on the $v$ th subcarrier, $N_{c p}$ is the length of a cyclic prefix, and $s_{k n}[u]$ is the transmit signal at the $u$ th time index. The received signal of the $m$ th antenna of the BS is given as

$$
y_{m}[u] \triangleq \sum_{k=1}^{K} \sum_{n=1}^{N}\left(\sqrt{P_{k}} h_{m k n} s_{k n}[u]\right)+z_{m}[u],
$$

where the power of $s_{k n}[u]$ is $\mathbb{E}\left[\left|\sqrt{P_{k}} s_{k n}[u]\right|^{2}\right]=1, P_{k}$ is the transmit power of the $k$ th user, $h_{m k n}$ is the channel response between the $n$th antenna of the $k$ th user and the $m$ th antenna of the BS, and $z_{m}[u] \sim C N\left(0, \sigma_{U L}^{2}\right)$ is the thermal noise.

With the assumption of identical low-resolution ADCs, the in-phase and quadrature components of the received signal of the $m$ th antenna are quantized by the ADCs of $b$-bit resolution as follows,

$$
q_{m}[u] \triangleq Q\left(\mathcal{R}\left(y_{m}[u]\right)\right)+j Q\left(\operatorname{Im}\left(y_{m}[u]\right)\right),
$$

where $Q($.) represents quantization; $\mathcal{R}($.$) and \mathcal{J}($.) denote the real and imaginary parts, respectively. The quantization through the ADCs worsens the accuracy of channel estimation and deteriorates the system performance. The quantization noise is given as,

$$
e_{m}[u]=q_{m}[u]-y_{m}[u] .
$$

The receiver removes the cyclic prefix and put into a discrete Fourier transform (DFT) block. The signal on the $v$ th subcarrier is then given as,

$$
Y_{m}[v]=\sum_{k=1}^{K} \sum_{n=1}^{N} \sqrt{P_{k}} H_{m k n}[v] S_{k n}[v]+\left(Z_{m}[v]+E_{m}[v]\right),
$$

where,

$$
\begin{aligned}
& S_{k n}[v]=\frac{1}{\sqrt{N_{d f t}}} \sum_{u=0}^{N_{d f t}-1} s_{k n}[u] e^{-j 2 \pi u v / N_{d f t}}, \\
& H_{m k n}[v]=h_{m k n} e^{-j 2 \pi v / N_{d f t}}, \\
& E_{m}[v]=\frac{1}{\sqrt{N_{d f t}}} \sum_{u=0}^{N_{d f f}-1} e_{m}[u] e^{-j 2 \pi u v / N_{d f t}},
\end{aligned}
$$

and $\mathrm{Z}_{m}[v] \sim C N\left(0, \sigma_{U L}^{2}\right)$ is the thermal noise that is given by,

$$
Z_{m}[v]=\frac{1}{\sqrt{N_{d f t}}} \sum_{u=0}^{N_{d f f}-1} z_{m}[u] e^{-j 2 \pi u v / N_{d f f}} .
$$

\subsection{Channel estimation}

Least-square (LS) estimation is employed for channel estimation [6] as follows. Suppose that data symbols are mapped at the subcarriers, which are the $(\lambda)$ th to the $\left(\lambda+N_{s c}-1\right)$ th subcarrier, where $N_{s c}$ is the number of active subcarriers, and a channel response is estimated for each subcarrier. The transmit active subcarriers are divided as $B=\left(N_{s c} / V\right)$ blocks in frequency domain, where $V$ is the number of subcarriers in one block. $N_{\text {sym }}=L / V$ OFDM symbols are required when the uplink pilots for $L$ streams are inserted at each $V$ subcarriers to estimate channel responses. 
The channel in the $b$ th block is estimated during a channel estimation period. The subcarrier index, $\{v\}$, in the $b$ th block is from $(b-1) V+\lambda$ to $b V$. The estimated channel for the $b$ th block is

$$
\hat{H}_{m k n}^{b}=\frac{1}{V} \sum_{v=(b-1) V+\lambda}^{b V-1+\lambda}\left(S_{k n}[v]\right)^{-1} Y_{m}[v],
$$

where $S_{k n}[v]$ and $Y_{m}[v]$ are the orthogonal sequence and the received signal on the $v$ th subcarrier, respectively.

\subsection{Uplink performance}

In the uplink, an ergodic rate per arbitrary user $k$ is

$$
C_{k}^{U L}=\sum_{n=1}^{N} \frac{1}{B V} \sum_{b=1}^{B} \sum_{v=(b-1) V+\lambda}^{b V-1+\lambda} \log _{2}\left(1+\operatorname{SINR}_{k n}^{v}\right),
$$

where

$$
\begin{aligned}
& \operatorname{SINR}_{k n}^{v}=\frac{\left|\mathbb{E}\left\{\mathbf{W}_{k n}^{b} \mathbf{H}_{k n}[v]\right\}\right|^{2}}{\sum_{n=1}^{N} \sum_{\substack{i=1 \\
i \neq k}}^{K} \mathbb{E}\left\{\left|\mathbf{W}_{k n}^{b} \mathbf{H}_{i n}[v]\right|^{2}\right\}+\left.\sum_{\substack{j=1 \\
j \neq n}}^{N} \mathbb{E}\left\{\mathbf{W}_{k n}^{b} \mathbf{H}_{k j}[v]\right\}\right|^{2}+\sigma_{U L}^{\prime}{ }^{2}}, \\
& \sigma_{U L}^{\prime}{ }^{2}=\mathbb{E}\left\{\left\|\mathbf{W}_{k n}^{b}\right\|^{2}\right\} \sigma_{U L}^{2}+\left|\mathbb{E}\left\{\mathbf{W}_{k n}^{b} \mathbf{E}_{m}[v]\right\}\right|^{2},
\end{aligned}
$$

$\mathbf{W}_{k n}^{b}$ is the maximum ratio combining or minimum mean square error detection coefficients in the vector form, they are given as MRC:

$$
\mathbf{W}_{k n}^{b}=\left(\hat{\mathbf{H}}_{k n}^{b}\right)^{\mathrm{H}}=\left(\left[\begin{array}{c}
\hat{\mathrm{H}}_{1 k n}^{b} \\
\vdots \\
\hat{\mathrm{H}}_{M k n}^{b}
\end{array}\right]\right)^{\mathrm{H}},
$$

Or MMSE:

$$
\mathbf{W}_{k n}^{b}=\left(\left(\left(\hat{\mathbf{H}}^{b}\right)\left(\hat{\mathbf{H}}^{b}\right)^{\mathrm{H}}+\sigma_{U L}^{\prime}{ }^{2} \mathbf{I}\right)^{-1}\left(\hat{\mathbf{H}}_{k n}^{b}\right)\right)^{\mathrm{H}},
$$

where $\hat{\mathbf{H}}^{b}$ and $\hat{\mathbf{H}}_{k n}^{b}$ are the channel estimation response including estimation error, in the $b$-th block and $\mathbf{H}_{k n}[v]$ is the channel responses between the $n$th antenna of the $k$ th user and the base station antennas in the vector form,

$$
\mathbf{H}_{i j}[v]=\left[\begin{array}{c}
\mathrm{H}_{1 i j}[v] \\
\vdots \\
\mathrm{H}_{M i j}[v]
\end{array}\right],
$$

$\mathbf{E}[v]$ is the quantization noise vector given as

$$
\mathbf{E}[v]=\left[\begin{array}{c}
\mathrm{E}_{1}[v] \\
\vdots \\
\mathrm{E}_{M}[v]
\end{array}\right] .
$$

Finally, ${\sigma_{U L}^{\prime}}^{2}$ is the sum of the variance of the thermal noise and the quantization noise.

\subsection{Proposed ADC design}

In the proposed $\mathrm{ADC}$, the quantization range is given as

$$
\begin{aligned}
& A^{\max }=C * \mathbb{E}\left(\left|y_{m}[u]\right|\right), \\
& \Delta=\frac{2 A^{\max }}{2^{R}},
\end{aligned}
$$


antenna of the BS, and $\Delta$ is the quantization step-size. The quantization range is adjusted via the coefficient $C$ and the quantization converts the real input signal to a real-valued output, $r_{i}$, for $i=1,2, \ldots, 2^{R}$. The $i$ th output value after ADCs is defined as

$$
q_{i}=\left(-\frac{2^{R}}{2}-\frac{1}{2}+i\right) \Delta .
$$

Thus, its value is adjusted for suppressing extra noise. Since each antenna element of the massive MIMO system generally receives a weak signal, the amplitude of which is smaller than that of the thermal noise and limiting the quantization range improves the signal quality after combining in the receiver.

\section{Numerical results}

\subsection{Simulation conditions}

Table I. Simulation conditions

\begin{tabular}{l|c}
\hline \multicolumn{1}{c|}{ Bandwidth } & $20 \mathrm{MHz} / \mathrm{RB}$ \\
\hline Number of Antennas & $\begin{array}{c}\text { Massive MIMO: } M=128,1024 \\
(=\text { horizontal } 8,64 \times \text { vertical 16), } \\
\text { Each user: } N=2\end{array}$ \\
(= horizontal $1 \times$ vertical 2),
\end{tabular}

Computer simulation conditions are presented in Table I. The massive MIMO BS receives signals with $M=128$ or 1024 antenna elements. In MU transmission, there are eight users (each user with $N_{u}=2$ antennas) that communicate with the BS simultaneously. The antenna spacing is $0.5 \lambda$ at the BS and $1.0 \lambda$ at each user, where $\lambda$ is the wavelength. The number of active subcarriers is 1200 while the DFT size is 2048 . The number of blocks is 150 and the number of subcarriers per block is 8 . Since the number of symbols for channel estimation is two, the number of signal streams whose channel responses can be estimated with Zad-off Chu sequences as orthogonal sequences is 16. As a channel model, i.i.d. Rayleigh fading is assumed. The resolution of ADCs is selected from 1, 2, 3, 4, or infinite bits. System throughput is the total rate of eight users. The number of trials for each plot is 10000 .

\subsection{System performance}

Performance of MRC and MMSE with low-resolution ADCs under different amounts of antennas is presented in Fig. 2. As shown in Figs. 2(a) and (b), when 
the number of antennas is 128 , the performance with infinite ADCs is not only limited by MUI, but also the over-clipping of the original signal deteriorates the channel estimation accuracy. With infinite ADCs, MMSE detection is better due to MUI elimination.

Because of a limited number of BS antennas, low-resolution ADCs has produced severe bit error rate (BER) in a TDD OFDM system. In a massive MIMO system, a large number of BS antennas can be equipped to reduce the BER. However, the MMSE signal detection algorithm involves matrix inversion with large complexity (especially in massive MIMO). MRC can avoid complicated matrix inversion. In Fig. 2(c) and (d), they are clear that the performance of MRC with 1024 antennas is as good as MMSE. MRC can achieve even better performance than that of MMSE with 1, 2, 3-bit ADCs.

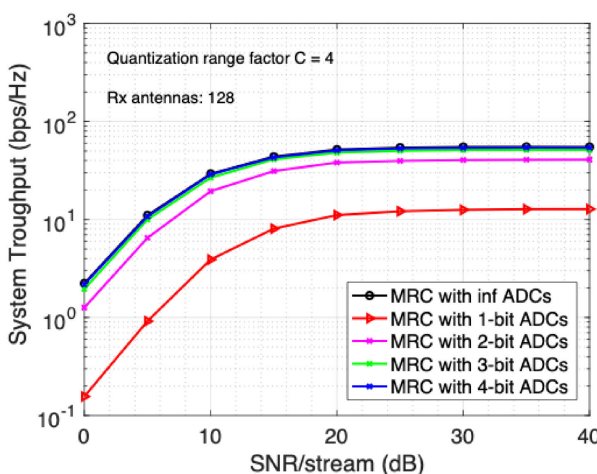

(a) $\mathrm{MRC}$

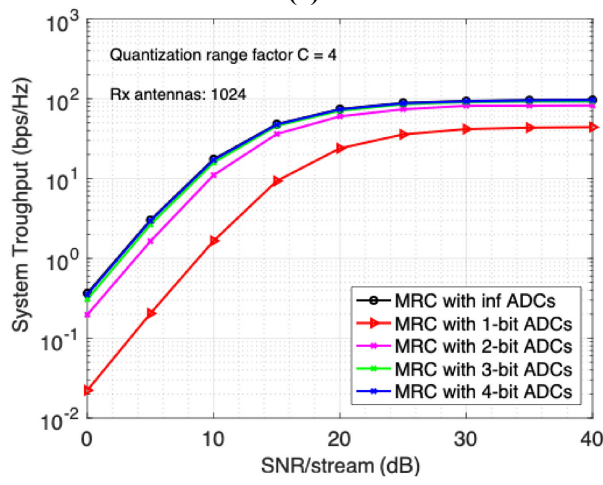

(c) $\mathrm{MRC}$

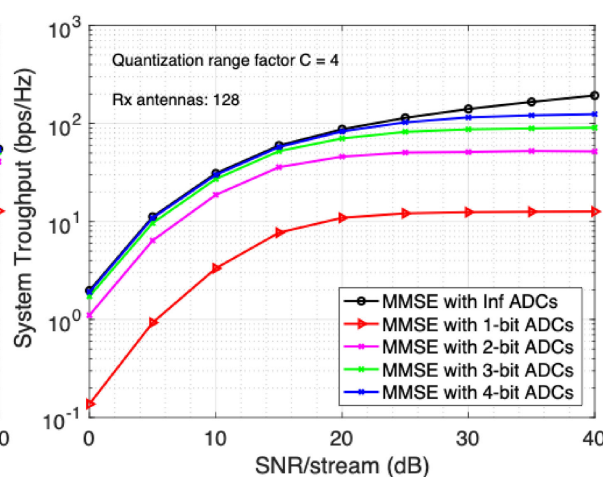

(b) MMSE

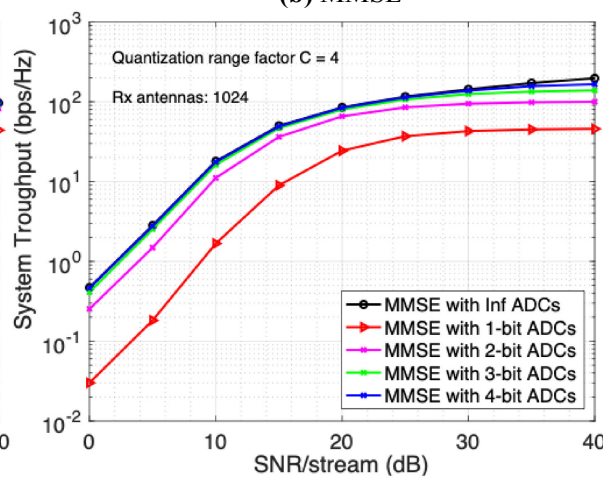

(d) MMSE

Fig. 2. Performance of MRC and MMSE with low-resolution ADCs

\section{Conclusions}

The different signal detection algorithms used by the uplink receiver in a multi-user massive MIMO system are compared. Under a large number of BS antennas, MMSE with higher resolution ADCs is better than MRC, although the matrix inversion operation demands a large amount of computational complexity. On the other hand, MRC can achieve equivalent or even better performance, especially with low-resolution ADCs. The system throughput realized in MRC with 2-bit ADCs is almost the same as that with infinite resolution ADCs and MRC can achieve better performance than MMSE with 1, 2, 3-bit ADCs. In addition, it requires less computational complexity. Therefore, MRC is more suitable as the number of BS antenna elements increases in a full-digital massive MIMO with low-resolution ADCs. 